\title{
Theoretical asthma self-management program for Taiwanese adolescents with self-efficacy, outcome- expectancy, health behavior, and asthma symptoms: A randomized controlled trial
}

\section{Tzu-Jung Tseng}

Chang Gung University of Science and Technology

Chiung-Jung (Jo) Wu ( $\nabla$ cwu@usc.edu.au)

University of the Sunshine Coast https://orcid.org/0000-0001-6512-9049

Anne M Chang

Queensland University of Technology

Research article

Keywords: Adolescent; Self-efficacy, Asthma self-management, Randomised controlled trial, Outcomeexpectancy

Posted Date: August 9th, 2019

DOl: https://doi.org/10.21203/rs.2.12004/v1

License: (c) (1) This work is licensed under a Creative Commons Attribution 4.0 International License.

Read Full License 


\section{Abstract}

Background: The asthma prevalence of Taiwanese adolescents is continued to rise and affecting sufferers' daily life. With physical and psychological changes in adolescents, managing asthma can be quite challenges for them and could lead to negative social, emotional and physical impacts if not well managed. Literature found that adolescents with high levels of self-efficacy are more likely to be involved in prevention and management activities. The aim of this study was to develop an effective asthma selfmanagement program by taking account for adolescents' developmental stages and based on Bandura's self-efficacy model for this group of young people. Methods: A randomised controlled trial was used to evaluate effects of the newly developed Asthma Self-management Program for Taiwanese adolescents. The outcomes were self-efficacy, outcome-expectancy, asthma prevention and self-management behaviour, and controlling asthma symptoms. Translation and back-translation process were used for original tools in English. Good consistent reliabilities of Chinese versions tools were identified. A t-test or Mann-Whiney $U$ test was employed to determine any mean differences of outcome variables between groups overtime. Results: A sample of 83 patients completed $(n=43$ in control group, $n=40$ in experimental group) the study. The mean age was 14.3 years $(S D=1.85)$, two-third of the participants were males. Seventy-six percent $(n=68)$ had a mild to intermittent level of asthma severity. Significant improvements were found on self-efficacy, $t=-2.75$; outcome-expectancy, $U=646$, prevention behaviours, $\mathrm{t}=-3.62$, and management behaviours, $\mathrm{t}=-3.19$, but non-significant results for the outcome of asthma control, $U=716$. Conclusions: The results provide evidence to support the efficacy of the asthma selfmanagement program based on the self-efficacy model that has taken considerations of developmental phases. The findings indicated the self-management program improved adolescents' self-confidence in carrying out asthma prevention and management behaviours for effectively manage their asthma symptoms. Future clinical practice should consider delivering an educational program using multifaceted and interactive learning strategies.

\section{Background}

Asthma is a chronic condition with an estimated 358.2 million sufferers worldwide [1]. More than $20 \%$ of adolescents aged 10-14 years were diagnosed with asthma in North America, United Kingdom, and Australia, and those in this age group have more mortality or disability than in any age groups [2]. The biological, psychological, and socio-emotional changes adolescents are undergoing during their development have considerable impacts on asthma management for this group [3, 4]. Adolescents with chronic diseases are reported to have a high frequency of non-adherence to treatment regimens [5]. Similar findings with an increased trend on asthma cases and intention no-adherence for Taiwanese adolescents have been experienced [2]. According to the National Health Insurance Research Database in Taiwan in 2017, the prevalence of adolescents with asthma continues increased (from $12.99 \%$ to $22.02 \%$ ) [6]. Many of them take no actions when experiencing asthma attacks [7], results in poor asthma outcomes $[8,9]$. 
Evidence showed that self-management programs guided by a theoretical framework provide more solid basis and lead to effective outcomes [10] Self-efficacy model includes efficacy-expectation (performance accomplishment, vicarious experience, verbal persuasion, and physiological information) and outcomeexpectation (what outcome people expect from engaging in a particular behaviour) [11]. Asthma selfmanagement programs (ASMPs) based on the self-efficacy model have been widely used [12-14], however such theoretical based intervention programs using a rigorous research design have not been investigated in Taiwan [15-17].

Furthermore, improving the effectiveness of asthma self-management programs for adolescents is imperative not only providing them with knowledge, but also considering their physical and psychosocial developmental stages [18]. For example, adolescents with asthma may tend to make themselves unattached to their asthma symptoms; they thought these could be a normal phenomenon or progression, and the symptoms would disappear by nature, without realising the symptoms would be a serious of asthma exacerbation [19]. Many adolescents did not use medications while the symptoms occurred due to the embarrassment of taking medications in front of their friends [19]. Some adolescents may feel that their parent or teacher do not care much about their asthma conditions, as they continue surrounded by parent's persistent smoking, and/or teachers' disapprovals for stopping physical activities when expecting an trigger of asthma exacerbation [20]. Thus, special developmental considerations should be made. This highlights an urgent need to effectively address asthma management.

\section{Aim}

This study aimed to develop and evaluate efficacy of the asthma self-management program (ASMP) for adolescents in Taiwan.

\section{Methods}

\section{Design}

A randomized, two-arm and parallel-group controlled trial was used. The primary outcome was asthma self-efficacy and secondary outcomes were outcome-expectancy, asthma prevention and management behaviours, as well as controlling asthma symptoms.

\section{Sample size}

A target sample of 112 patients was initially estimated based on the medium effect size (.5) [21] of selfefficacy mean scores, with $19.1 \%$ attrition rates [22]. With time restraints, 90 patients were subsequently recruited when reaching the effect size of.58. The statistical significance was set at.05.

Inclusion criteria included being diagnosed with asthma and receiving treatment, 12 to 18 years old, able to converse in Chinese, using an owned mobile phone. Exclusion criteria were being diagnosed with a 
cognitive deficiency. This study was conducted in a more than 1000-bed tertiary hospital located in northern Taiwan.

\section{Randomisation}

As outlined in our protocol paper [23], randomization was undertaken using a computerized random number, generated by a statistician who was independent to this study. Each participant was blindly allocated to either control or experimental group. Participants in the experimental group received the ASMP in addition to the usual care. The standard asthma care (usual care) for participants in the control group consisted of asthma care information based on the National Health Insurance Institute Administration Ministry of Health and Welfare guidelines, including information for improving symptoms management, such as asthma medications, monitoring and symptoms management [24], and it was provided by a registered nurse in the outpatient clinics.

\section{Intervention}

The ASMP for Taiwanese adolescents was developed based on Bandura's self-efficacy model [11]. The content of the ASMP as described on our protocol paper [23] were developed using the National Asthma Educational and Prevention Program Experts Panel Report-3 guideline (EPR-3) [25]. The guideline consisted of environmental factors and comorbid conditions that affect asthma, education for a partnership in care, medication, assessing and monitoring asthma severity and asthma control. Validity of the ASMP contents in Chinese was determined by a panel of experts who have reached $100 \%$ agreements [26].

The modes of delivery adapted from a multi-components of self-management program for patients with chronic diseases [27]. The ASMP contained three face-to-face sessions, followed by two short text messages and a telephone call. A booklet titled "Living with Asthma Together" including "My Asthma Action Plan" was provided at the first face-to-face meeting for recording participants' goals for asthma management in week 1 , followed by discussing about their feeling and concerns about asthma symptoms in week 2 face-to-face meeting. At week 3 , the ASMP session was focused on problem solving skills of asthma exacerbation. Two text messages were sent weekly after each meeting for 4 weeks. A telephone call was made at week 4 to inquire how the participant was progressing. An example of applying the self-efficacy model and the contents of the ASMP is presented in Figure 1 [28].

\section{Insert Figure 1 in here}

\section{Data collection}

The outcome measures of self-efficacy, outcome-expectancy, prevention and management behaviours, as well as the symptoms of asthma were collected at T1 (baseline) and T2 (week 4). The procedure of data collection was presented in our protocol paper [23]. Data collection was undertaken by a trained research assistant who was blinded to the study protocol. See Figure 2. 


\section{Measures}

Outcomes of asthma self-efficacy, outcome-expectancy, asthma prevention and management behaviours, and controlling asthma symptoms were measured by Asthma Self-Efficacy Index [12], Outcomeexpectancy [29]. Asthma Self-management index [12] and the Asthma Control Test [30] at T1 and T2. The original instruments for asthma self-efficacy, outcome-expectancy, and asthma self-management behaviours (prevention and management) were only available in English. With permissions obtained, translating these English versions into Chinese by translation and back-translation process [23]. The Cronbach's alphas to support the reliabilities of the Chinese versions of the Asthma Self-efficacy index and Outcome-expectancy were 0.83 and 0.88 , respectively. The asthma Prevention Index and the Asthma Management Index to measure asthma self-management were 0.73 and 0.67 respectively. The Cronbach's alphas of asthma control test was 0.84 [28].

\section{Data analysis}

The homogeneity of demographic and outcomes variables was examined prior to data analysis. Data were analysed using SPSS version 21. Chi-square test was used to determine differences between groups for demographic categorical variables of sex, family smoking experience, and asthma severity levels, whereas independent t-test was used for age and years of asthma between groups. The mean differences of self-efficacy and prevention and management behaviour were examined by t-test; Mann-Whitney Utest was applied for outcome-expectancy and asthma control test. The level of significance was set at $p<.05$.

\section{Ethical Approvals}

The study was approved by the participating hospital Institutional Review Boards (CGMFIRB No1023787B) and university Human Research Ethics Committee (Ethics No140000006). Informed consents were obtained from adolescents and their parents/guardians. The researcher explained the purpose of the study; what they did during the study; what their rights, benefits, and risks participation this study to adolescents and their parents separately. If adolescents and their parents agreed to participate this study, they were asked to provide written assent and consents. Participants were also ensured their participation was voluntary. All data were saved on the university secured drive and limited access permitted to only authorised researchers to ensure the privacy and confidentiality of data.

\section{Results}

\section{Sample Characteristics}

A total of 90 patients were recruited, seven of them withdrew from this study due to a busy school schedule. There were no statistical differences in age, gender, family smoking, severity of asthma and 
outcome variables between completed and discontinued participants. The remaining 83 participants were randomly allocated to experimental $(n=40)$ and control $(n=43)$ groups.

The mean age was 14.3 years $(S D=1.85)$, majority of the participants were males $(n=52)$. Most of adolescents had a mild to intermittent level of asthma severity $(n=68)$. All the participants denied smoking, but only half of their parents were non-smokers.

\section{Outcomes}

\section{Asthma self-efficacy}

The mean asthma self-efficacy at T2 for the experimental group was 110 points ( $S D=21.7$ ), while the mean was $91.7(S D=26.6)$ for the control group. There were differences 18.6 points $(S D=20.2)$ from T2 to $\mathrm{T} 1 \mathrm{for}$ the experimental group. Although the mean asthma self-efficacy at T2 for the control group also increased from T1, it was to a lesser extent of 6.65 points $(S D=19.2)$. An independent t-test indicated there was a significant difference between the two groups for the change from T1 to T2 self-efficacy mean level, $t(81)=-2.75, p<.05$. This finding indicated that participants in the experimental group had significant improvement in asthma self-efficacy (see Table 1).

\section{Outcome-expectancy}

The median outcome-expectancy scores for the control group at T2 was 64 (minimum $=14$, maximum $=$ 80 ), however, the median outcome-expectancy score for the experimental group was 70 (range from 46 to 80). Mann-Whitney $U$ test indicated that there was a significant difference between the two groups regarding the change in outcome-expectancy scores, $U=646, p<.05$, The improvements in outcomeexpectancy was greater for the experimental group ( 5 points) than for the control group ( 2 points) (see Table 2).

\section{Asthma prevention and management behaviors}

The scores of the asthma preventive behaviour for the experimental group was $24.6(S D=4.74)$ at T2. There increased 3.9 point $(S D=4.55)$ from $\mathrm{T} 1$ to $\mathrm{T} 2$. The mean of preventive behaviour for the control group was $21.3(S D=4.99)$ at T2. There also increased but only by 0.7 points $(S D=3.67)$ from $T 1$ to T2. Results of the statistical analysis revealed there was a significant difference between the two groups for the change from T1 to T2 preventive behaviour mean score, $t(81)=-3.62, p<.05$. Adolescents with asthma who received the asthma self-management program performed more asthma preventive behaviours in order to control their asthma symptoms compared to adolescents with asthma who received the usual asthma care. 
The mean of asthma management behaviour for the experimental group at T2 was $19.9(S D=4.63)$, while the mean of management behaviour for the control group was $17.8(S D=4.82)$. The mean asthma management behaviour score for the experimental group had increased by 2.55 points $(S D=3.96)$ from T1 to T2, but the mean asthma management behaviour score for the control group had dropped by 0.12 points $(S D=3.66)$. The change in management behaviours was significantly greater for the experimental group than for the control group, $t(81)=-3.19, p<.05$. Participants who received the asthma selfmanagement program carry out more asthma management behaviours compared to participants who received the standard asthma care when they experienced asthma attacks (see Table 1).

\section{Asthma symptoms}

The difference in median Asthma Control Test (ACT) from T1 to T2 was 1.0 for two groups. The MannWhitney $U$ indicated there was no significant difference between the two groups regarding the change in the ACT, $U=716(p>.05)$. These findings indicate that the change in the asthma control test scores from T1 to T2 was not significantly different between groups (see Table 2).

Insert Table 2 here

\section{Discussion}

The significant effects of the ASMP were found in self-efficacy, outcome-expectancy, and management behaviours, but not on asthma symptoms. The ASMP has taken accounted for adolescents' developmental stages to further advance the significant results. Previous studies showed that adolescents often denied their disease, non-adhered to asthma management recommendations, as they may worry about medications side effects, felt embarrassed about using an inhaler in front of their peers $[5,31,32]$. Adolescents could be seen as "not tough", feared to be kept away from their peers, therefore they declined to make their peers aware of their asthma conditions and missing any necessary support [18]. Furthermore, adolescents would prefer to feel respected, have their own autonomy to make all relevant decisions [4]. The ASMP was implemented by valuing adolescents' prior asthma knowledge and experience, they were more willing to carry out their own asthma self-care plan. The communications were perceived by the participants to be open-minded, non-judgemental, which led to a trusting relationship. Subsequently convincing patients to take in charge and make good effort of carrying their own asthma self-care plan in better managing plan their asthma symptoms.

The findings demonstrated the benefits of an intervention program guided by the self-efficacy model on the health outcomes for patients with chronic diseases such as asthma [13]. The results are consistent with a systematic review recommending of using all self-efficacy strategies to optimise the effectiveness of self-management programs for chronic diseases such as asthma [10]. This study was based on Bandura's self-efficacy model through performance accomplishment, vicarious experience, verbal 
persuasion, and self-evaluation of physiological and emotional states [11]. Performance accomplishment is the strongest source of increasing self-efficacy; discussing desires and achievable goals were applied to the ASMP. Mastery on asthma self-management skills and vicarious experience by using role models in the booklet were included. Verbal persuasion further enhancing self-efficacy was performed by a phone call and text messages. Finally, physiological and emotional states were aware by feelings of having asthma flare-up. In contrast, in other studies [14] where self-management intervention without applying or explicitly illustrating all four sources for enhancing self-efficacy would have less effective. Thus, the findings support the need of integrating four sources of information to increase patients' self-efficacy to an asthma self-management program for adolescents.

The significant improvements in outcome-expectancy levels have highlighted the importance of positive beliefs in performing asthma self-management prevention and management behaviours. The ASMP helped adolescents with asthma to balance both their needs and effective control of their asthma symptoms. Explaining asthma treatment plans to adolescents' enhanced adherence and lead to less asthma symptoms [33,34]. Furthermore, greater outcome-expectancy improvements may be due to provide an individual discussion at face-to-face sessions about adolescents' peer relationships, hence promoting their willingness to perform asthma preventive behaviours [18].

The significantly improved asthma prevention and management behaviours is related to people who have high self-confidence and outcome-expectancy levels [11]. Evidence from previous research supports self-efficacy as the strongest predictor for health promoting behaviours in adolescents [34]. Previous studies have reported that adolescents with asthma and high levels of self-efficacy had better asthma adherence [35], as well as self-confidence in coping with barriers when performing their asthma self-care [36]. It may be that participants who received the asthma self-management program had a better understanding of the purpose of prevention behaviours and felt confident in carrying out such behaviours. Consequently participants may have then been more willing to perform asthma self-care activities [37].

Improved asthma management behaviours could be possibly enhanced by in-depth and cumulated discussions. For example, participants in the ASMP were more self-reflecting their own asthma symptoms after relief inhaler usage and asking for help with worsening asthma symptoms. These results were unlike those of previous studies, which found that adolescents with asthma did not treat or manage their breathing problems when they had an asthma attack [34,38]. These findings demonstrated that the ASMP made positive progressing of managing asthma flare-ups. Moreover, a written asthma action plan was provided to participants for enabling them to take more achievable steps in managing an asthma flare-up [39, 40].

The statistical insignificant Asthma Control Test (ACT) scores could be due to participants who were at less severity levels of asthma levels (mild intermittent to moderate). This finding is similar to a study showed more obvious effectiveness on moderate and severe asthma patients [41]. Another possible rationale could be due to the seasonal factors. Moist and cold weather may have induced more severe 
asthma symptoms $[42,43]$, however, the ASMP was conducted in low humidity summer season in Taiwan. Therefore, a longitudinal study of the ASMP will be needed.

\section{Limitations}

The limitation of follow up time after implementing the ASMP is identified. Data collection with all seasons will provide evidence for long-term effect.

\section{Conclusions}

This study has addressed gaps in past research of not using a theoretical model to guide the development of an ASMP led to inconsistent results. The features of the ASMP included taking account of a key behaviour measurement of outcome-expectancy, adolescents' developmental characteristics and needs. The positive results demonstrated the effects of the ASMP in asthma self-efficacy, selfmanagement, outcome-expectancy, prevention and management behaviours. The ASMP has also highlighted the great potential benefits of using multifaceted and interactive learning strategies into an educational program.

\section{Abbreviations}

- ASMP: Asthma Self-Management Program

- ACT: Asthma Control Test

\section{Declarations}

Ethics approval and consent to participant: Full ethics approvals from the participating hospital Institutional Review Boards (CGMFIRB No102-3787B) and university Human Research Ethics Committee (Ethics No140000006) have been obtained. All adolescents and their parents were provided with a copy of the assent, informed consents and study information.

Consent for publication: Not applicable

Availability of data and materials: All data supporting the findings in this article is contained within the manuscript.

Competing interests: The authors declare that they have no competing interests.

Funding: This study was supported by the Postgraduate Research awards Scholarship and Student Allocation funding from Queensland University of Technology, Brisbane, Australia. The funder has no role in the study design, data collection, analysis and interpretation of data, in manuscript preparation and submitting the article for publication. 
Author Contributions: Study conception and design: TJT, C-JW, AMC. Data collection: TJT, C-JW, AMC. Data analysis: TJT, C-JW, AMC. Manuscript drafts of all versions: TJT, C-JW; Manuscripts appraisals and editing: TJT, C-JW. ICMJE criteria for authorship read and met: TJT, C-JW, AMC. Agree with manuscript results, conclusions and submissions: TJT, C-JW, AMC.

Acknowledgements: Not applicable

\section{References}

1.Soriano JB, Abajobir AA, Abate KH, Abera SF, Agrawal A, Ahmed MB, et al. Global, regional, and national deaths, prevalence, disability-adjusted life years, and years lived with disability for chronic obstructive pulmonary disease and asthma, 1990-2015: a systematic analysis for the Global Burden of Disease Study 2015. The Lancet Respiratory Medicine. 2017;5(9):691-706.

2.Hwang C-Y, Chen Y-J, Lin M-W, Chen T-J, Chu S-Y, Chen C-C, et al. Prevalence of Atopic Dermatitis, Allergic Rhinitis and Asthma in Taiwan: A National Study 2000 to 2007. Acta Derm Venereol. 2010;90(6):589-94.

3.Edgecombe K, Latter s, Peters s, Roberts G. Health experiences of adolescents with uncontrolled severe asthma. Archives Disesase in Childhood. 2010;95: (12):985-91.

4.Bitsko MJ, Everhart RS, Rubin BK. The adolescent with asthma. Paediatric Respiratory Reviews. 2014;15:146-53.

5.Koster ES, Philbert D, de Vries TW, van Dijk L, Bouvy ML. "I just forget to take it": asthma selfmanagement needs and preferences in adolescents. The Journal of Asthma. 2015;52(8):831-7.

6.Ma Y-C, Lin C-C, Li C-I, Chiang J-H, Li T-C, Lin J-G. Time-trend analysis of prevalence, incidence and traditional Chinese medicine use among children with asthma: a population-based study. Journal of Public Health. 2016;38(3):e263-e71.

7. Health Promotion Administration Ministry of Health and Welfare. 2013 National health interview survey in Taiwan 2013 [Available from: http://www.nhri.org.tw.

8.Mosnaim GS, Pappalardo AA, Resnick SE, Codispoti CD, Bandi S, Nackers L, et al. Behavioral Interventions to Improve Asthma Outcomes for Adolescents: A Systematic Review. The Journal of Allergy and Clinical Immunology: In Practice. 2016;4(1):130-41.

9.Blake KV. Improving adherence to asthma medications: current knowledge and future perspectives. Current opinion in pulmonary medicine. 2017;23(1):62-70.

10. Lycett HJ, Raebel EM, Wildman EK, Guitart J, Kenny T, Sherlock J-P, et al. Theory-Based Digital Interventions to Improve Asthma Self-Management Outcomes: Systematic Review. Journal Of Medical Internet Research. 2018;20(12):e293-e. 
11.Bandura A. Self-efficacy in changing societies. Cambridge; New York: Cambridge University Press; 1995. xv, 334 p. p.

12.Bruzzese JM, Unikel LH, Shrout PE, Klein RG. Youth and Parent Versions of the Asthma-Related Anxiety Scale: Development and Initial Testing. Pediatric allergy, immunology, and pulmonology. 2011;24(2):95-105.

13.Wu C-J, Chang AM. Application of a theoretical framework to foster a cardiac-diabetes selfmanagement programme. International Nursing Review. 2014;61(3):336-43.

14.Korpershoek C, van der Bijl J, Hafsteinsdóttir TB. Self-efficacy and its influence on recovery of patients with stroke: a systematic review. Journal of advanced nursing. 2011;67(9):1876-94.

15.Lin HC, Chiang LC, Wen TN, Yeh KW, Huang JL. Development of online diary and self-management system on e-Healthcare for asthmatic children in Taiwan. Computer methods and programs in biomedicine. 2014;116(3):299-310.

16.Maa SH, Chang YC, Chou CL, Ho SC, Sheng TF, Macdonald K, et al. Evaluation of the feasibility of a school-based asthma management programme in Taiwan. Journal of clinical nursing. 2010;19(1718):2415-23.

17.Yang B, Chen Y, Chiang B, Chang Y. Effects of Nursing Instruction on Asthma Knowledge and Quality of Life in Schoolchildren with Asthma. The Journal of Nursing Research. 2005;13(3):174-83.

18. Holley S, Morris R, Knibb R, Latter S, Liossi C, Mitchell F, et al. Barriers and facilitators to asthma selfmanagement in adolescents: A systematic review of qualitative and quantitative studies. Pediatric Pulmonology. 2017;52(4):430-42.

19.Stewart M, Letourneau N, Masuda JR, Anderson S, Cicutto L, McGhan S, et al. Support needs and preferences of young adolescents with asthma and allergies: "just no one really seems to understand". Journal Pediatric Nursing 2012;27(5):479-90.

20.Sattoe JNT, Bal MI, Roelofs PDDM, Bal R, Miedema HS, van Staa A. Self-management interventions for young people with chronic conditions: A systematic overview. Patient Education and Counseling. 2015;98(6):704-15.

21.Reddan MC, Lindquist MA, Wager TD. Effect Size Estimation in NeuroimagingEffect Size Estimation in NeuroimagingEffect Size Estimation in Neuroimaging. JAMA Psychiatry. 2017;74(3):207-8.

22.Bruzzese JM, Sheares BJ, Vincent EJ, Du Y, Sadeghi H, Levison MJ, et al. Effects of a school-based intervention for urban adolescents with asthma. A controlled trial. American journal of respiratory and critical care medicine. 2011;183(8):998-1006. 
23.Tseng T-J, Chang AM, Wu C-J. A randomized controlled trial of an asthma self-management program for adolescents in Taiwan: A study protocol. Contemporary Clinical Trials Communications. 2017;8(Supplement C):122-6.

24.Tzeng LF, Chiang LC, Hsueh KC, Ma WF, Fu LS. A preliminary study to evaluate a patient-centred asthma education programme on parental control of home environment and asthma signs and symptoms in children with moderate-to-severe asthma. Journal of clinical nursing. 2010;19(9-10):142433.

25.NHLBI. Expert Panel on the Diagnosis and Management of Asthma. Expert Panel Report 3: Guidelines for the Diagnosis and Management of Asthma. US: National Heart, Lung, and Blood Institute; 2008 December 2008.

26.Polit DF, Beck CT, Owen SV. Is the CVI an acceptable indicator of content validity? Appraisal and recommendations. Research in nursing \& health. 2007;30(4):459-67.

27.Wu C-J, Atherton JJ, Maclsaac RJ, Courtney M, Chang AM, Thompson DR, et al. Effectiveness of the cardiac-diabetes transcare program: protocol for a randomised controlled trial. BMC health services research. 2017;17(1):109.

28.Tseng T-J. Asthma self-management program for adolescents in Taiwan. Bribsane: Queensland University of Technology; 2016.

29.Zebracki K, Drotar D. Outcome expectancy and self-efficacy in adolescent asthma self-management. Children's Health Care. 2004;33(2):133-49.

30.Schatz M, Sorkness CA, Li JT, Marcus P, Murray JJ, Nathan RA, et al. Asthma Control Test: Reliability, validity, and responsiveness in patients not previously followed by asthma specialists. The Journal of allergy and clinical immunology. 2006;117(3):549-56.

31.Blanchard M, Morris J, Birrell E, Stephens-Reicher J, Third A, Burns J. National Young people and Asthma Suvery: Issues and Opportunities in the Health and Wellbing of Young people Living With Asthma Young and Well CRC, Melbourne.; 2014.

32.Peláez S, Lamontagne AJ, Collin J, Gauthier A, Grad RM, Blais L, et al. Patients' perspective of barriers and facilitators to taking long-term controller medication for asthma: a novel taxonomy. BMC pulmonary medicine. 2015;15:42-.

33.Wigfield A, Eccles JS. Expectancy-Value Theory of Achievement Motivation. Contemporary Educational Psychology. 2000;25(1):68-81.

34.Velsor-Friedrich B, Vlasses F, Moberley J, Coover L. Talking with teens about asthma management. The Journal of school nursing 2004;20(3):140-8. 
35.Bruzzese JM, Idalski Carcone A, Lam P, Ellis DA, Naar-King S. Adherence to Asthma Medication Regimens in Urban African American Adolescents: Application of Self-Determination Theory. Health psychology: official journal of the Division of Health Psychology, American Psychological Association. 2013.

36.Rhee H, Belyea MJ, Ciurzynski S, Brasch J. Barriers to asthma self-management in adolescents: Relationships to psychosocial factors. Pediatric pulmonology. 2009;44(2):183-91.

37.Clerisme-Beaty EM, Bartlett SJ, Teague WG, Lima J, Irvin CG, Cohen R, et al. The Madison Avenue effect: How drug presentation style influences adherence and outcome in patients with asthma. Journal of Allergy and Clinical Immunology. 2011;127(2):406-11.

38.Jonsson M, Egmar A-C, Hallner E, Kull I. Experiences of living with asthma-a focus group study with adolescents and parents of children with asthma. Journal of Asthma. 2014;51(2):185-92.

39.Villa-Roel C, Voaklander B, Ospina MB, Nikel T, Campbell S, Rowe BH. Effectiveness of written action plans for acute asthma: A systematic review. J Asthma. 2018;55(2):188-95.

40.Kelso JM. Do Written Asthma Action Plans Improve Outcomes? Pediatric allergy, immunology, and pulmonology. 2016;29(1):2-5.

41.Saito J, Sato S, Fukuhara A, Sato Y, Nikaido T, Inokoshi Y, et al. Association of Asthma Education with Asthma Control Evaluated by Asthma Control Test, FEV1, and Fractional Exhaled Nitric Oxide. Journal of Asthma. 2013;50(1):97-102.

42.Covar RA, Szefler SJ, Zeiger RS, Sorkness CA, Moss M, Mauger DT, et al. Factors associated with asthma exacerbations during a long-term clinical trial of controller medications in children. Journal of Allergy and Clinical Immunology. 2008;122(4):741-7.e4.

43.Wang Y-C, Lin Y-K. Temperature effects on outpatient visits of respiratory diseases, asthma, and chronic airway obstruction in Taiwan. International Journal of Biometeorology. 2015;59(7):815-25.

\section{Tables}

Table 1. Comparison of T1 and T2 differences in self-efficacy, prevention and management behaviors for two groups $(n=83)$ 


\begin{tabular}{|c|c|c|c|c|c|c|}
\hline & \multicolumn{2}{|c|}{ Experimental } & \multicolumn{2}{|c|}{ Control } & & \\
\hline & T1 & T2 & T1 & T2 & & \\
\hline & $M$ & $M$ & $M$ & $M$ & & \\
\hline & $(S D)$ & $(S D)$ & $(S D)$ & $(S D)$ & & \\
\hline Self-efficacy & 90.2 & $\begin{array}{c}110 \\
(226)\end{array}$ & $\begin{array}{l}83.6 \\
(227)\end{array}$ & 91.7 & $12.049(-20.5$, & $-2.75 *$ \\
\hline$(0-140)$ & & & & & & \\
\hline $\begin{array}{l}\text { Prevention } \\
\text { behaviors }\end{array}$ & $\begin{array}{l}20.4 \\
(5.2)\end{array}$ & $\begin{array}{l}24.6 \\
(4.74)\end{array}$ & $\begin{array}{c}20.3 \\
(5.45)\end{array}$ & $\begin{array}{c}21.3 \\
(4.99)\end{array}$ & $\begin{array}{c}3.20(-1.39 \\
-5.01)\end{array}$ & $-3.51 *$ \\
\hline$(0-33)$ & & & & & & \\
\hline Management & $\begin{array}{l}17.2 \\
172)\end{array}$ & $\begin{array}{l}19.9 \\
1962)\end{array}$ & 17.8 & 17.7 & $2.67(-4.34,-1.0)$ & $-3.18^{*}$ \\
\hline 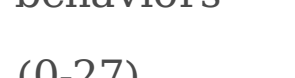 & & & $(4.04)$ & $(4.82)$ & & \\
\hline
\end{tabular}

$$
* P<0.05
$$

Table 2. Comparison of T1 and T2 differences in outcome-expectancy and asthma symptoms for two groups $(\mathrm{n}=83)$

\begin{tabular}{|c|c|c|c|c|c|}
\hline & & Group & & & Mann-Whitney \\
\hline Variables & Experim & ntal & & & \\
\hline & T1 & T2 & T1 & T2 & \\
\hline & Median (min- & Median & Median & Median & \\
\hline & & $\begin{array}{l}(\min - \\
\max )\end{array}$ & $(\min -$ & $(\min -$ & \\
\hline Outcome- & 64 & 70 & 63 & 64 & 6428* \\
\hline$(0-80)$ & $(21-80)$ & $(46-80)$ & $(9-80)$ & $(14-80)$ & \\
\hline Asthma Symptoms & 22 & 23 & 22 & 23 & 716 \\
\hline$(0-25)$ & $(8-25)$ & $(15-25)$ & $(12-25)$ & $(1425)$ & \\
\hline
\end{tabular}

$* P<0.05$ 


\section{Figures}

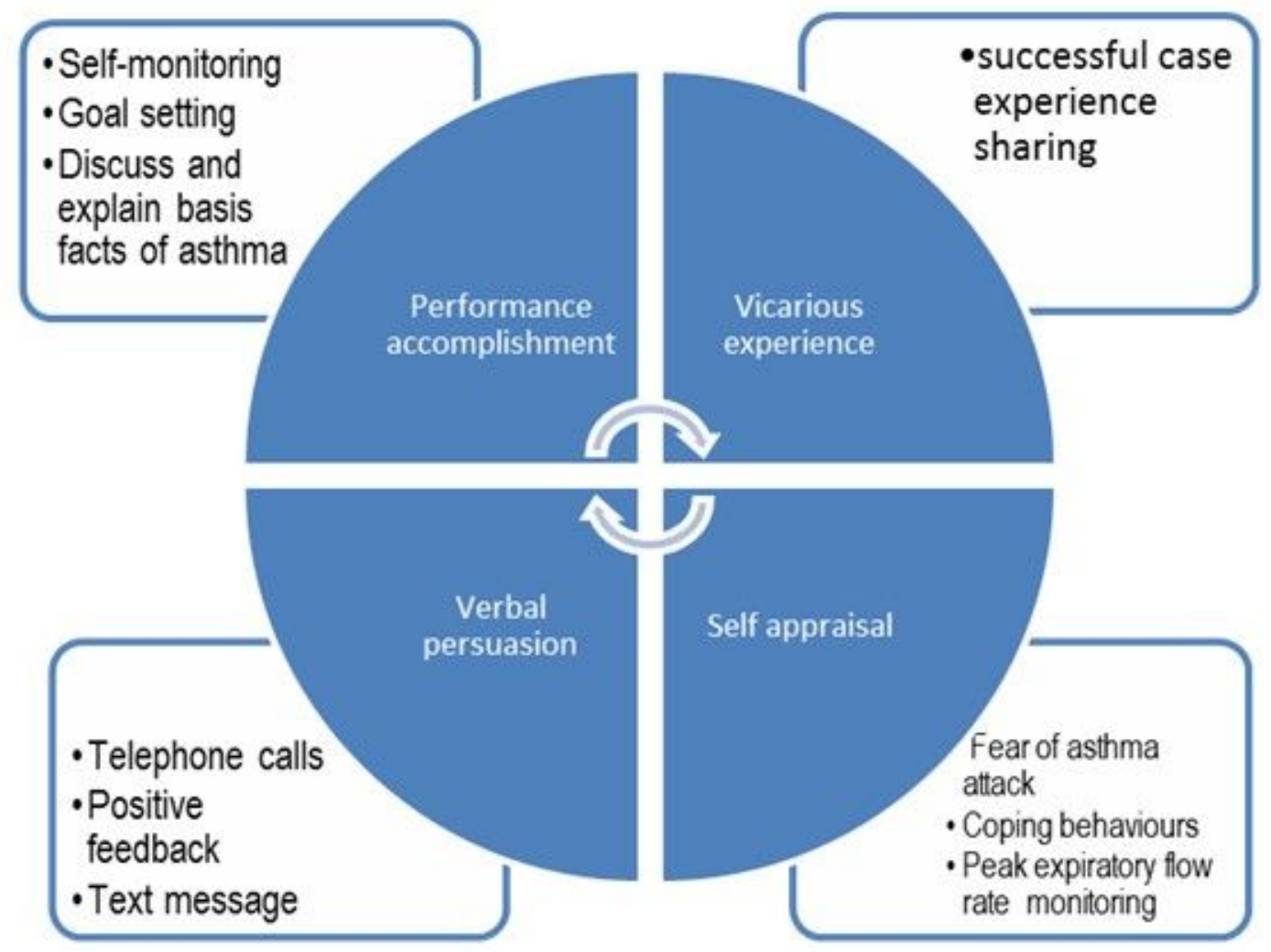

Figure 1 


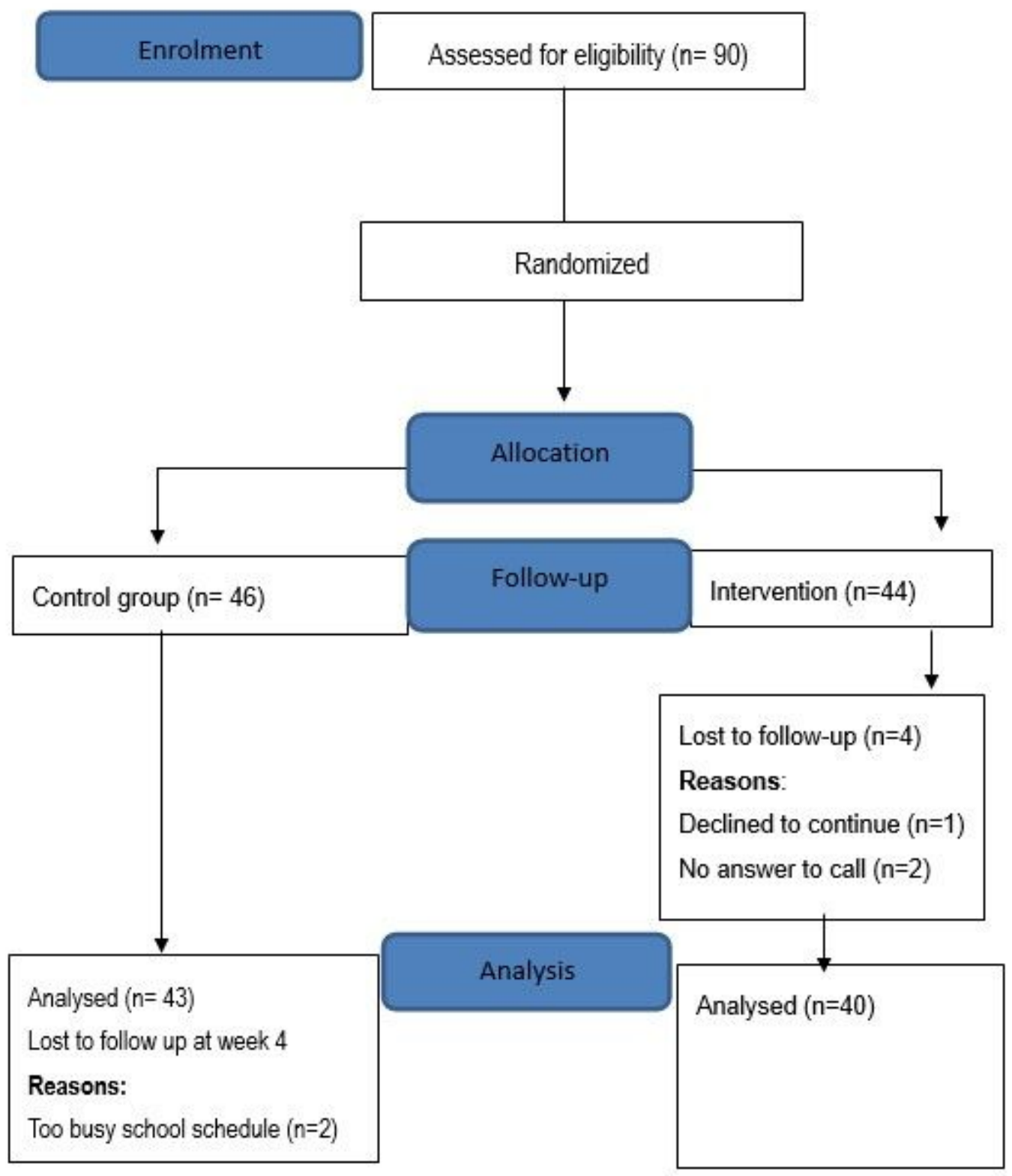

Figure 2 